\title{
Targeting Axon Growth from Neuronal Transplants along Preformed Guidance Pathways in the Adult CNS
}

\author{
Kristine S. Ziemba, ${ }^{1}$ Nagarathnamma Chaudhry, ${ }^{1,2}$ Alexander G. Rabchevsky, ${ }^{1}$ Ying Jin, ${ }^{1}$ and George M. Smith ${ }^{1}$ \\ ${ }^{1}$ Spinal Cord and Brain Injury Research Center, and Department of Physiology, University of Kentucky, Lexington, Kentucky 40536, and ${ }^{2}$ Department of \\ Biological Sciences, Hunter College, New York, New York, 10021
}

To re-establish neuronal circuits lost after CNS injury, transplanted neurons must be able to extend axons toward their appropriate targets. Such growth is highly restricted within the adult CNS attributable to the expression of inhibitory molecules and general lack of guidance cues to direct axon growth. This environment typically induces random patterns of growth and aberrant innervation, if growth occurs at all. To target the growth of axons from neuronal transplants, we are using viral vectors to create guidance pathways before neuronal transplantation. In this study, we transplanted postnatal rat dorsal root ganglia neurons into the corpus callosum of adult rats. Replication-incompetent adenoviruses encoding growth or guidance factors were injected along the desired pathway 1 week before cell transplantation, allowing time for sufficient protein expression by host glial cells. With expression of nerve growth factor (NGF) and basic fibroblast growth factor, sensory axons were able to grow along the corpus callosum, across the midline, and toward an NGF-expressing target in either the contralateral striatum or cortex: a distance of 7-8 mm including a $90^{\circ}$ turn from white matter into gray matter. Furthermore, expression of semaphorin $3 \mathrm{~A}$ slightly dorsal and lateral to the turning point increased the number of axons turning into the striatal target. These results show that judicious expression of neuron-specific chemoattractant and chemorepellant molecules using viral vectors can support and target axon growth from neuronal transplants in the adult CNS.

Key words: neurotrophins; transplantation; sensory neurons; semaphorin 3A; axon guidance; gene transfer

\section{Introduction}

CNS damage caused by disease or injury may be repaired by neural transplantation, but only if certain obstacles are overcome. Finding a safe, reliable source of cells for transplantation is both a scientific and an ethical challenge, but that is just the first step. Once cells are prepared for transplantation, a sufficient number of neurons must survive the transplantation procedure and extend fibers, sometimes over long distances, to reach appropriate targets and replace damaged circuitry. Transplantation experiments have produced promising results in animal models of spinal cord injury, amyotrophic lateral sclerosis, epilepsy, stroke, Parkinson's disease and Huntington's disease, and several clinical trials have already been conducted for these disorders (Bjorklund and Lindvall, 2000; Isacson et al., 2003). In most cases, cellreplacement therapy is limited to local effects on host circuitry, thus only enhancing the effectiveness of endogenous circuits without reconstructing damaged ones. Overall, this limits the ability of the transplant to restore function, which remains susceptible to disease progression. These neuronal transplants fail to reestablish endogenous circuits because axons are unable to ex-

Received Aug. 21, 2007; revised 0ct. 31, 2007; accepted Nov. 27, 2007.

This work was supported by National Institute of Neurological Disease and Stroke Grant R01 NS38126 and the Kentucky Spinal Cord and Head Injury Research Trust. We thank Melody King for help with axon quantification, and Drs. Steve Estus and Gabrielle Curinga for critical review of this manuscript.

Correspondence should be addressed to Dr. George M. Smith, Department of Physiology, University of Kentucky, 800 Rose Street, Lexington, KY 40536-0298. E-mail: gmsmith@uky.edu.

DOI:10.1523/JNEUROSCI.3819-07.2008

Copyright $\odot 2008$ Society for Neuroscience $\quad$ 0270-6474/08/280340-09\$15.00/0 tend over long distances in the adult CNS. This limitation is most likely attributable to two related issues: a lack of growthpromoting molecules and guidance cues in the adult host tissue, and the presence of inhibitory molecules, especially after injury (Aubert et al., 1995; Sandvig et al., 2004; Domeniconi and Filbin, 2005; Schwab et al., 2005).

Although fetal or stem cell-derived neurons used for transplant studies have a high intrinsic growth potential (Cai et al., 2001; Goldberg et al., 2002) their ability to reconstruct damaged circuits is very low, partially because of the inhibitory nature and lack of guidance cues within the adult CNS. Embryonic or adult dorsal root ganglion (DRG) neurons, when transplanted into the corpus callosum of adult hosts, extended axons for several millimeters along the tract only if scarring produced by transplantation was absent (Davies et al., 1994, 1997). If the microinjections were too large and produced a scar containing chondroitin sulfate proteoglycan (CSPG), growth was inhibited. We have adapted this model using large transplants that produce some scar formation to investigate the ability of growth factors and guidance molecules to enhance and direct the growth of DRG neurons from the transplantation site across the corpus callosum to contralateral striatal or cortical targets. Likely candidates to enhance and direct growth of these axons are nerve growth factor (NGF) and/or fibroblast growth factor-2 (FGF2), which were shown previously to induce robust sprouting and regeneration of DRG neurons within the spinal cord (Romero et al., 2000, 2001). Using a similar strategy, adenovirus encoding NGF or FGF2 was used to create a preformed growth pathway extending across the 
corpus callosum and turning into the contralateral cortex or striatum.

\section{Materials and Methods}

Construction of adenoviral vectors. Replicationdefective, temperature-sensitive recombinant adenoviruses encoding NGF, FGF2, semaphorin $3 \mathrm{~A}$, and green fluorescent protein (GFP) were constructed as described previously (He et al., 1998; Romero and Smith, 1998; Tang et al., 2004). All plaque-purified adenoviruses were examined for replication-competent adenoviruses by PCR and were amplified and purified by double cesium chloride gradient ultracentrifugation. The absolute concentration of viral particles was determined by optical absorbency, and the concentration of infectious particles (plaque-forming units) was quantified by viral hexon protein expression in infected human embryonic kidney 293 (HEK293) cells using the Adeno-X Rapid Titer kit (BD Biosciences, Franklin Lakes, NJ). Expression of each protein by virus-infected U373 cells was confirmed by Western blot $72 \mathrm{~h}$ after transfection as described previously (Romero et al., 2000; Tang et al., 2004).

Adenovirus injections. Adult female Sprague Dawley rats (retired breeders; Harlan, Indianapolis, IN) were used for all experiments. Animals were maintained under conditions of controlled light and temperature, with food and water available ad libitum. On the day of surgery, rats were anesthetized with a mixture of ketamine $(67 \mathrm{mg} / \mathrm{kg}$, i.p.) and xylazine $(6.7 \mathrm{mg} / \mathrm{kg}$, i.p. $)$ and placed into stereotactic frames. Just before, and the day after surgeries, rats were given intraperitoneal injections of monoclonal antibodies to block the T-cell receptors CD4 (W3/25, 50 $\mu \mathrm{g})$ and $\mathrm{CD} 45$ (OX-22, $50 \mu \mathrm{g})$, to suppress any immune response to the adenovirus (Ad) (Romero and Smith, 1998). For comparisons of guidance pathways, animals were randomly assigned to one of four treatment groups: (1) Ad-FGF2 along the guidance pathway, Ad-NGF at the target, (2) combination Ad-FGF2/Ad-NGF along the pathway, Ad-NGF at the target, (3) Ad-GFP along the pathway, Ad-NGF at the target, or (4) Ad-GFP along the pathway and at the target (no NGF). Guidance pathways were created by injecting each adenovirus every $1 \mathrm{~mm}$ from a transplant site in the left lateral corpus callosum to a $90^{\circ}$ turn into the contralateral striatum (target). GFP showed that this injection pattern resulted in continuous distribution of virus along the corpus callosum. Using bregma as a landmark, holes were drilled into the skull to allow injections at the following coordinates (all $0.3 \mu \mathrm{m}$ caudal, depths relative to dura): transplant site, $+2.8 \mathrm{~mm}$ lateral (left side), $-3.0 \mathrm{~mm}$ deep; pathway within the corpus callosum, $\pm 2.5 \mathrm{~mm}$ lateral, $-3.0 \mathrm{~mm}$ deep, $\pm 1.5 \mathrm{~mm}$ lateral, $-2.9 \mathrm{~mm}$ deep and $\pm 0.7 \mathrm{~mm}$ lateral, $3.2 \mathrm{~mm}$ deep; pathway into striatum at -2.5 $\mathrm{mm}$ lateral (right side), depths of $3.5 \mathrm{~mm}$ and $4.0 \mathrm{~mm}$; striatal target, $-2.5 \mathrm{~mm}$ lateral, $4.5 \mathrm{~mm}$ deep. For the semaphorin experiment, all animals received pathways of Ad-FGF2/Ad-NGF and striatal NGF targets, and then one-half of the animals received two $0.4 \mu \mathrm{l}$ injections of Ad-semaphorin $3 \mathrm{~A} 1 \mathrm{~mm}$ dorsolateral from the pathway turning point at $3.2 \mathrm{~mm}$ lateral, $2.2 \mathrm{~mm}$ deep, and $3.6 \mathrm{~mm}$ lateral, $2.6 \mathrm{~mm}$ deep. For the cortical target experiment, the striatal path and target were replaced with injections at $-2.5 \mathrm{~mm}$ lateral, and 2.5, 2.0, and $1.5 \mathrm{~mm}$ deep. Adenovirus concentrations were $5 \times 10^{6} \mathrm{pfu} / \mu \mathrm{l}$, except for combination Ad-FGF2/ Ad-NGF injections, where the Ad-NGF concentration was reduced to $1 \times 10^{6} \mathrm{pfu} / \mu \mathrm{l}$ for a $5: 1$ ratio of Ad-FGF2 to Ad-NGF. Injection volumes ranged between $0.2 \mu \mathrm{l}$ (Ad-NGF at transplant site) and $2.0 \mu \mathrm{l}$ (Ad-NGF at striatal target), and increased along the pathway to create a gradient effect; however, the importance of gradient formation to direct growth is
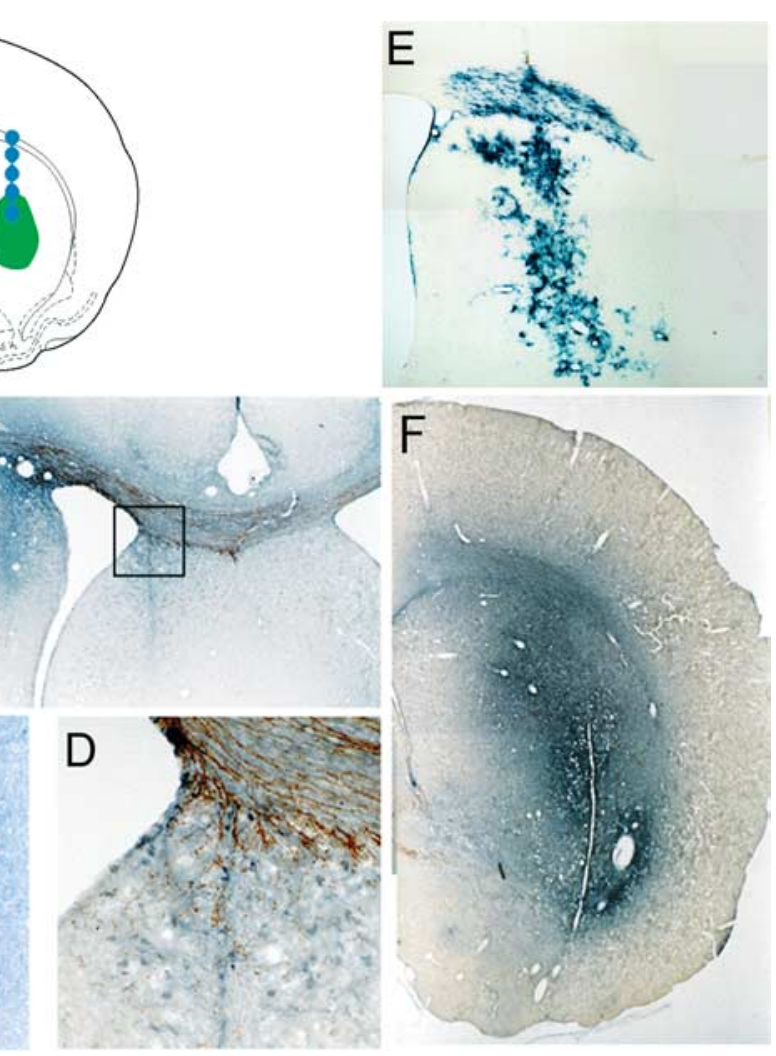

Figure 1. Confirmation of expression of NGF and FGF2 after adenoviral injections into the corpus callosum and striatum. $\boldsymbol{A}$

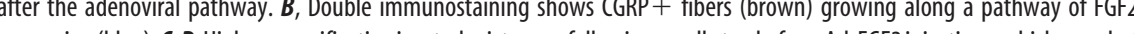
the corpus callosum. $\boldsymbol{E}$, Beta-galactosidase staining shows the location and density of transfected cells $7 \mathrm{~d}$ after injection of LacZ/Ad. F, Immunostaining for NGF shows widespread expression in the target striatum and up into the external capsule.

questionable. Volumes were injected at a rate of $0.4 \mu \mathrm{l} / \mathrm{min}$ using a $10 \mu \mathrm{l}$ Hamilton syringe and a 30 -gauge beveled needle, and the needle remained in place for $2 \mathrm{~min}$ at the end of each injection.

$D R G$ isolation and transplantation. One week after adenovirus injections, DRG neurons were isolated from postnatal day 1 (P1) or P2 Sprague Dawley rat pups (timed-pregnant dams from Harlan). Each pup was quickly decapitated and DRGs were removed using sterile Dumont forceps and placed into Hank's buffer containing 1\% collagenase, kept on ice until all dissections were complete. After a $20 \mathrm{~min}$ incubation at $37^{\circ} \mathrm{C}$, DRGs were washed then trypsinized for $10 \mathrm{~min}$ at $37^{\circ} \mathrm{C}$, treated with DNase and washed twice more with $10 \%$ fetal bovine serum (FBS) in DMEM, then triturated to disperse the ganglia into a cell suspension. Cells were then plated out in 10\% FBS/DMEM containing $50 \mathrm{ng} / \mathrm{ml} \mathrm{NGF}$ for $45 \mathrm{~min}$ at $37^{\circ} \mathrm{C}$ to allow adhesion of Schwann cells. Cells remaining in suspension were then spun down and washed in N2-supplemented media three times to eliminate FBS. The number of live neurons per microliter was determined by treatment with trypan blue and counting on a hemocytometer. The final cell suspension was supplemented with NGF $(50 \mathrm{ng} / \mathrm{ml})$ and kept on ice until transplant (up to $3 \mathrm{~h}$ total). Rats previously injected with adenovirus were reanesthetized and their skulls exposed again at the transplant site $(2.8 \mathrm{~mm}$ lateral, $0.3 \mathrm{~mm}$ caudal to bregma). Three to five thousand DRG neurons were injected with a Hamilton syringe/30-guage needle in a volume of $<2 \mu \mathrm{l}(0.4 \mu \mathrm{l} / \mathrm{min})$ into the left corpus callosum, $3 \mathrm{~mm}$ below the dura. The needle was left in place for $10 \mathrm{~min}$, withdrawn $0.4 \mathrm{~mm}$, left for an additional $5 \mathrm{~min}$, and then slowly retracted the rest of the way.

Immunohistochemistry and quantification. Rats were killed 3 weeks after DRG transplants by pentobarbital overdose and perfusion with cold saline followed by $4 \%$ paraformaldehyde. Brains were removed and post- 
fixed in $4 \%$ paraformaldehyde overnight, then transferred to a $30 \%$ sucrose solution for $\sim 2 \mathrm{~d}$, allowing brains to sink to the bottom of the vials before cryosectioning. One in five coronal sections (30 $\mu \mathrm{m}$ thick) through the transplant area was immunostained using a rabbit primary antibody to calcitonin gene-related peptide (CGRP; 1:10,000 dilution; The Jackson Laboratory, Bar Harbor, ME), a biotinylated secondary antibody (goat anti-rabbit IgG, 1:600), and developed with a diaminobenzidine chromogen to visualize nociceptive neuronal cell bodies and axons. For each animal, three sections in the one in five series (150 $\mu \mathrm{m}$ apart) with the largest number of CGRP+ cells were quantified for transplant survival or axon growth along the pathway by a blinded observer. For cell survival, the total number of visible CGRP + cell bodies at the transplant site were manually counted at 200 times the total magnification and summed over the three sections. Axons were counted manually at 200 times the total magnification at the following points along the pathway: in the corpus callosum 1 and $2 \mathrm{~mm}$ from midline ipsilateral to transplant (midline $-1 \mathrm{~mm}$; midline $-2 \mathrm{~mm}$ ), in the corpus callosum 1 and 2 $\mathrm{mm}$ from midline contralateral to transplant (midline $+1 \mathrm{~mm}$, midline $+2 \mathrm{~mm}$ ), in the contralateral striatum $0.5 \mathrm{~mm}$ below the corpus callosum (c.c. $-0.5 \mathrm{~mm}$ ), and in the contralateral striatum $1 \mathrm{~mm}$ below the corpus callosum (c.c. $-1 \mathrm{~mm}$ ). To correct for differences in transplant size and survival, axon counts at the more distal path points were divided by a count closer to the transplant (midline $-2 \mathrm{~mm}$ or midline $-1 \mathrm{~mm}$ ). For quantification of turning success with and without semaphorin expression adjacent to the turn, animals with fewer than 10 axons at the turning decision point (average over three sections) were eliminated from the analysis. This led to elimination of three animals from each group. Axons were then counted at distances relative to the needle track from the Ad-NGF target injection: $200 \mu \mathrm{m}$ past the track in the corpus callosum, and $200 \mu \mathrm{m}$ below the corpus callosum in the striatum.

Statistical analyses. To analyze cell survival, t tests were used to compare groups without NGF at different time points and the +NGF group to each time point without NGF. To determine whether the molecule(s) expressed along the pathways affected the number of axons at different points along the pathway, nonparametric statistical tests were used to allow for smaller sample sizes (smallest group, $n=3$ ) and large variances that were observed within groups. A Kruskal-Wallis test was used to determine whether pathway composition affected axon count at each path point, and Mann-Whitney U tests were subsequently used to determine which pathways were significantly different at those points. For turning analysis with and without semaphorin, sample sizes were larger ( $n=8$ and 9, respectively) and an $F$ test confirmed equal variance, so a $t$ test assuming equal variance was used to compare those two groups. Differences were considered statistically significant if $p$ values were less than or equal to 0.05 .

\section{Results \\ Transplant survival requires Ad-NGF injection at transplant site}

Initial experiments followed the injection scheme depicted in Figure $1 A$, either with or without inclusion of a small-volume Ad-NGF injection at the transplantation site. Expression pathways were constructed by injecting Ad-FGF2 at several sites along the corpus callosum and down into the right striatum, where a larger volume ( $2 \mu \mathrm{l})$ of Ad-NGF was injected to create a target for growing axons. One week after virus injections, DRG neurons were isolated from P1-P2 rat pups and injected at the transplant site in the corpus callosum of the left hemisphere. Immunohistochemical staining in test animals 1 week after transplant confirmed that FGF2 was being expressed along the corpus callosum and that there was robust NGF expression in the target striatum (Fig. $1 B, F$ ). Staining for $\beta$-gal from animals injected with LacZ/Ad after identical injections parameters and viral titers as with $\mathrm{NGF} /$ Ad into striatal targets, shows the relative density and location of transfected cells (Fig. 1E). Furthermore, CGRP+ axons had grown along the expression pathway, some following needle tracks that overshot the corpus callosum during virus injections leading to FGF-2 expression in the underlying striatum (Fig. $1 B-$ $D)$. After allowing 2 weeks for axon growth, immunostaining of brain sections through the transplant site revealed good cell survival with NGF expression at the transplant site, but no visible CGRP + cells in animals without NGF at the transplant site. A subsequent time course study showed the demise of most transplanted neurons within the first few days after transplantation when no NGF support was provided (Fig. $2 A-D$ ). There was a significant drop in cell survival between days $1-3(n=4)$ and days $7-11(n=6 ; p=0.04)$, with no identifiable CGRP + cells at 2 weeks in those animals lacking NGF at the transplant site $(n=3)$. Expression of a small amount of NGF led to a significantly higher survival in the number of transplanted cells 2 weeks after transplantation when compared with those without NGF at any time point ( $p<0.05$ vs $1-3 \mathrm{~d}$ No NGF; $p<0.001$ vs $7-11 \mathrm{~d}$ No NGF). 
A statistical comparison could not be made at the 14-d time point because without NGF, the cell count was zero in all animals (no variance). Thus, our data support the role of NGF as both a survival-enhancing and axon-targeting molecule for postnatal nociceptive DRG neurons. In all subsequent animals in this study, a small volume of Ad-NGF was injected at the transplantation site along with the pathway injections 1 week before transplant to ensure survival of transplanted cells.

\section{The effects of FGF2, TGF- $\beta 1$, and L1 cell adhesion molecule as pathway substrates}

Previous studies by Davies et al. (1997) demonstrated axon growth along the corpus callosum only in animals receiving microtransplantation that did not induce expression of CSPG. If CSPG expression occurred, axons failed to extend very far within the corpus callosum. The first goal of these studies was to determine whether this potential barrier was present and if it could be overcome with growth factors. As demonstrated by Davies et al. (1997), we also observed increased expression of CSPG surrounding our relatively large DRG transplants. Because injury induces CSPG upregulation and these experiments required at least six injections within the corpus callosum, we also examined whether CSPG expression was increased within this tract after injection of adenovirus. Injections of equal concentrations of AdLacZ led to a relatively homogenous expression of $\beta$-galactosidase throughout the entire length of the corpus callosum (Fig. $3 A$ ). These injections resulted in robust expression of CSPG throughout the entire length of the corpus callosum, and along the individual needle tracks (Fig. 3). Axons failed to extend more than a few millimeters within this environment.

To examine the potential of various growth promoting molecules to influence axon growth out of the transplant, across the corpus callosum and toward a contralateral striatal target, LacZ, FGF2, L1-CAM, and transforming growth factor- $\beta 1$ (TGF- $\beta 1$ ) were injected to generate a pathway (Fig. 4). In these experiments, only pathways of FGF2 induced sufficient growth across the corpus callosum and into the contralateral striatum; in which about half of the axons turned and entered the striatum (Fig. 4B,D). Injections of LacZ (control) and FGF2/L1 combination induced limited axon growth across the corpus callosum most likely induced by target derived NGF (Fig. $4 A, E$ ). In all samples, axons grew only a short distance in the direction opposite of pathway where they stopped or looped around back toward the transplant (Fig. 4C). Interestingly, either $\mathrm{L} 1$ cell adhesion molecules or TGF- $\beta 1$ alone or in combination with FGF2 showed less axon growth out of transplants than controls (Fig. $4 F, G$ ). Both of these molecules are potentially involved in regulating the inhibitory nature of the environment. The cell adhesion molecule L1 forms part of the semaphorin $3 \mathrm{~A}$ receptor complex and enhances semaphorin mediated growth inhibition for these neurons (Castellani et al., 2000; Chaudhry et al., 2006). However, TGF- $\beta 1$ is a potent inducer of CSPG expression by astrocytes (Asher et al., 2000, Smith and Strutz, 2005) and led to very high levels of CSPG expression in these animals (data not shown). Thus, this model might be useful for examining the potential growth promoting or inhibitory nature of various guidance molecules on individual neuronal populations.

Axons do not cross midline without neurotrophin expression along pathway and/or at target site.

To determine whether a pathway expressing neurotrophins is necessary for growing nociceptive axons to reach the target area in the contralateral hemisphere, pathways were created by injec-
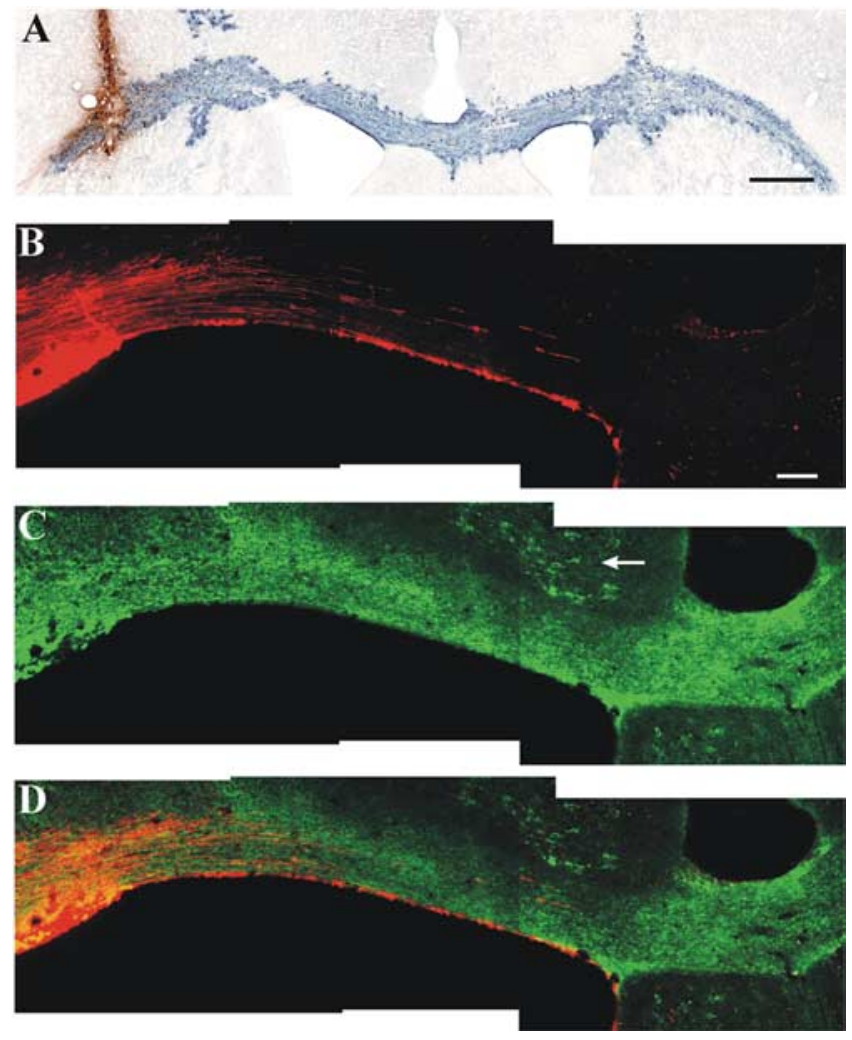

Figure 3. Injection of adenovirus increases expression of CSPG along the corpus callosum. $A$, Injection of LacZ/Ad at six locations along the corpus callosum shows good expression of the transgene (blue) and little axon growth from (GRP + DRG neurons (brown). $\boldsymbol{B}$, Higher magnification of an adjacent section showing diminished CGRP + axon growth after growing only several millimeters from transplant. $\boldsymbol{C}$, The entire length of the corpus callosum shows high expression of $(S P G$, with a few cells in the gray matter along the injection site also being positive for CSPG (arrow). D, Composite image shows axons grow poorly within this environment. Scale bars: $\boldsymbol{A}, 0.5 \mathrm{~mm} ; \boldsymbol{B}-\boldsymbol{D}, 100 \mu \mathrm{m}$.

tions of Ad-FGF2 or a combination of Ad-FGF2 and Ad-NGF (five to one ratio) and compared with control pathways created by injections of Ad-GFP. Every pathway included a small AdNGF injection at the transplant site to ensure transplant survival, and all but four GFP-pathway animals (GFP only) received AdNGF target injections in the contralateral striatum. Three weeks after transplantation, immunohistochemical staining for CGRP revealed that pathways expressing FGF2 $(n=4)$ or a combination of FGF2 and NGF $(n=8)$ supported robust, long-distance axon growth toward the target (Fig. $5 C, D$ ). All animals in these two groups had axons that grew at least $2 \mathrm{~mm}$ beyond the midline into the contralateral hemisphere, and 10 of 12 had some turning of axons from the corpus callosum into the target striatum (Fig. $5 I-M)$. In animals that received Ad-GFP injections only along the path and at the target $(n=4)$, no axons crossed the midline in the corpus callosum, and only two had axons growing $>1 \mathrm{~mm}$ away from the transplant site (Fig. $5 A, E, F$ ). Of three animals that had GFP pathways ending in an NGF target, only one had axon growth beyond the midline, with a few fibers turning into the contralateral striatum (Fig. $5 B, G, H$ ). In the other two animals, axon growth stopped at or before the midline. Expression of FGF2 and FGF2/NGF significantly increased the number of CGRP + fibers in the corpus callosum (Fig. $5 N$ ) at 1 and $2 \mathrm{~mm}$ past the midline (midline $+1 \mathrm{~mm}, p=0.006$; midline $+2 \mathrm{~mm}$, $p<0.01$ ) and at 0.5 and $1 \mathrm{~mm}$ below the corpus callosum in the target striatum (c.c. $-0.5 \mathrm{~mm}, p=0.03$; c.c. $-1 \mathrm{~mm}, p=0.05$ ) 

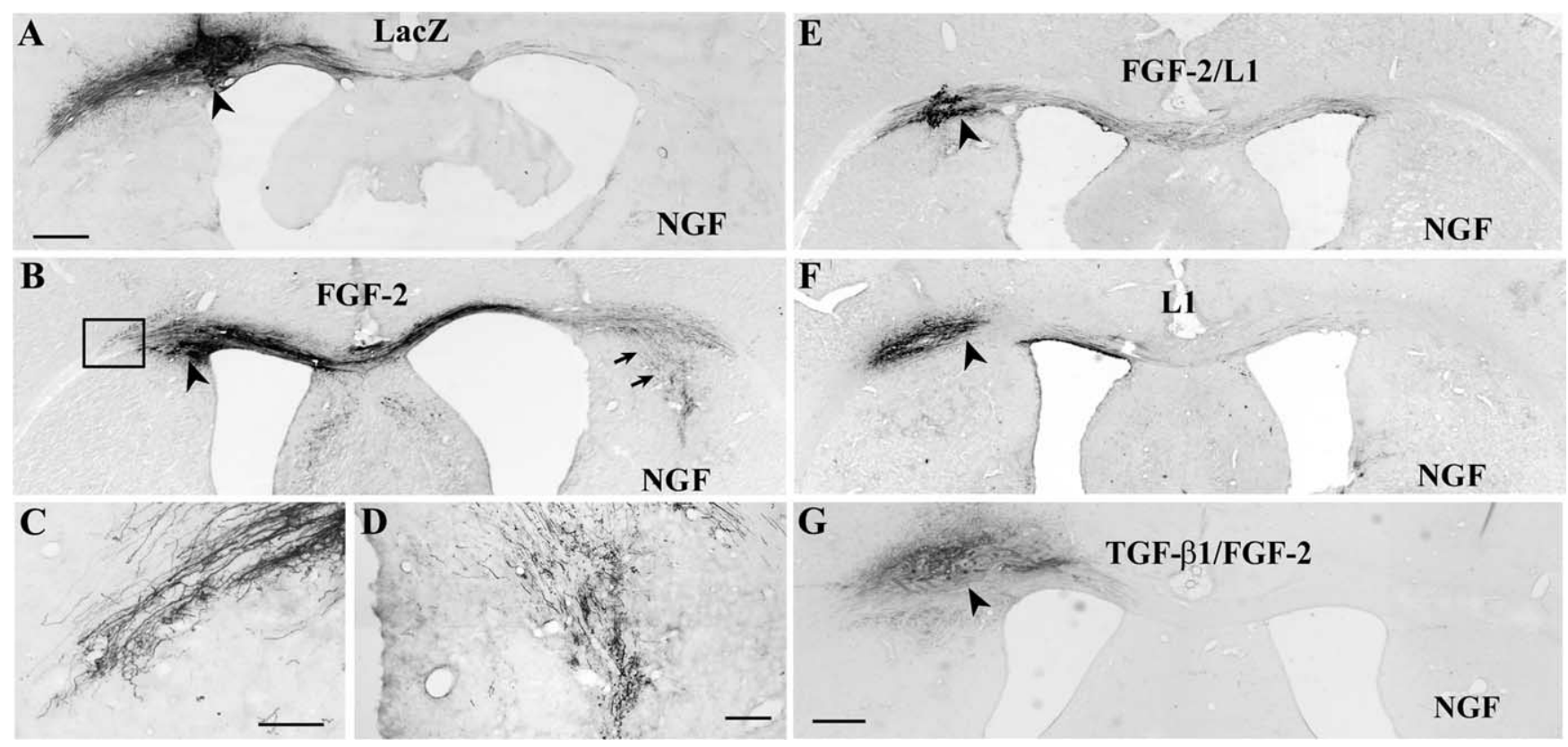

TGF- $\beta 1 /$ FGF-2

Figure 4. Expression of guidance cues can either be positive or negative regulators of axonal growth within the corpus callosum. Representative CGRP-immunostained sections show nociceptive axon growth 2 weeks after DRG neuron transplants. $\boldsymbol{A}$, LacZ expression pathway leading to an NGF striatal target supports little axon growth across the corpus callosum. $\boldsymbol{B}$, Pathway expression of FGF2 supports robust growth of axons across the corpus callosum into the contralateral external capsule; however, only about half of these axons turn and enter the striatum. $C$, Higher magnification of axons growing in the direction opposite to the expression pathway (arrowhead in $\boldsymbol{B}$ ). The vast majority of these axons either stop or make hairpin turns back toward the expression pathway. $\boldsymbol{D}$, Higher magnification of axons in $\boldsymbol{B}$ (double arrows) showing axons leaving the white matter tract and entering the striatum. $\boldsymbol{E}, \boldsymbol{F}$, Expression of the cell adhesion molecules $L 1$ does not support growth of axons along the corpus callosum $(\boldsymbol{F})$, and its coexpression with FGF2 reduces the growth supportive nature of FGF2 (E). TGF- $\beta 1$ even with the coexpression of FGF2 does not support axon growth along the pathway, and reduces it to less than LacZ controls. Scale bars: (in $\boldsymbol{A}) \boldsymbol{B}, \boldsymbol{E}-\boldsymbol{G}, 1 \mathrm{~mm} ; \boldsymbol{C}, \boldsymbol{D}, 200 \mu \mathrm{m}$

when compared with the GFP only group. Axon growth across the midline along either the FGF2 or FGF2 + NGF pathways were significantly higher (Mann-Whitney $U$ tests, $p<0.05$ ) at midline $+1 \mathrm{~mm}$ when compared with either of the GFP groups. Axon growth along the pathway at midline +2 and at $0.5 \mathrm{~mm}$ below the corpus callosum significantly increased (Mann-Whitney $U$ tests, $p<0.05$ ) between the GFP-only group and both FGF2expressing groups and between the GFP/NGF target group and the combination FGF2+NGF/NGF target group. The combination of FGF2+NGF pathway showed a significant increase in axon number (Mann-Whitney $U$ tests, $p<0.05$ ) along the entire pathway leading to the target when compared with GFP pathways either with or without NGF targets. This pathway resulted in $\sim 20 \%$ of the axons that crossed the midline to turn and enter the striatum to a depth of at least $1 \mathrm{~mm}$ (Fig. $5 \mathrm{~N}$ ). These results indicate that adenovirus-induced expression of FGF2 and NGF can enhance and direct sensory axonal growth from transplanted DRG neurons over distances of at least $6 \mathrm{~mm}$ overcoming an otherwise inhibitory environment.

Once in the contralateral hemisphere the pathway turned into the striatum. The bolus of NGF-Ad used to generate the striatal target diffused into the external capsule most likely preventing $\sim 50 \%$ of the axons from turning out of the white matter tract. We considered continued growth in white matter to be a targeting error.

\section{Expression of a chemorepulsive molecule adjacent to turn in pathway enhances axon turning}

Because many axons continued to grow in the corpus callosum beyond the striatal turn in the pathway (Fig. $5 J, L$ ), we next tested the hypothesis that expression of a chemorepulsive molecule adjacent to the turning point could increase the number of axons making the desired pathway choice. As shown in Figure 6A, the adenovirus injection scheme for this experiment included Ad-semaphorin $3 \mathrm{~A}$ (Ad-sema) injections $\sim 1 \mathrm{~mm}$ from the turn in the dorsolateral direction. Control animals received the same pathway and target injections (Ad-FGF2/NGF and Ad-NGF, respectively), but did not have Ad-sema injections. As in the previous experiments, DRG transplants were done 1 week after virus injections and animals were killed for histological analysis 3 weeks after transplantation. Coexpression of GFP along with semaphorin 3A shows the relative density and location of Sema $3 \mathrm{~A}$ expressing cell (green) within the cortex. The majority of these cells were localized above and slightly lateral to the turning of the CGRP+ axons (red) into the striatum (Fig. 6B). To compare axon turning in this experiment, animals in which CGRP+ fibers did not reach the pathway turning point in the contralateral hemisphere were eliminated from the analysis (three per group). In the remaining animals, an observer unaware of treatment counted CGRP+ fibers that continued past the target needle track in the corpus callosum and those that turned into the target striatum. The expression of semaphorin $3 \mathrm{~A}$ adjacent to the turning point resulted in a significant increase in the proportion of axons that make the desired turn, from $51 \%$ to $77 \%$ (no sema, $n=9$, mean \pm SEM, $51 \pm 7 \%$; with sema, $n=8,77 \pm 7 \%$; $p=0.02$ ) (Fig. $6 C-F$ ). These data support the hypothesis that adenovirus-mediated expression of chemorepulsive molecules can help to selectively target axon growth from transplanted neurons by reducing pathfinding errors.

\section{Cortical NGF expression leads to enhanced axon growth on brain surface}

An interesting observation in some animals was that some CGRP + fibers grew up into the cortex on the target side, follow- 

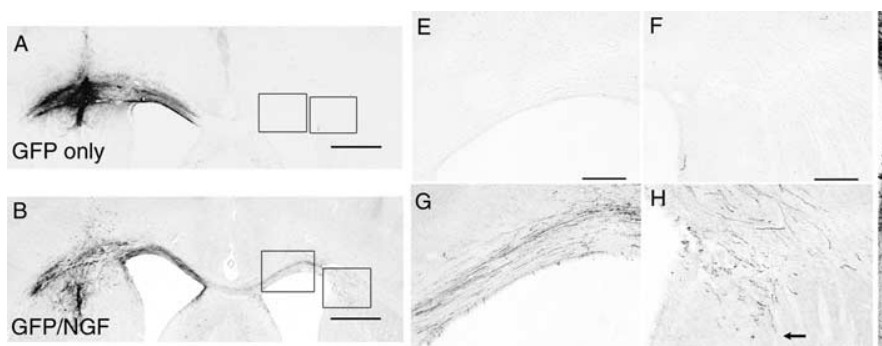

FGF/NGF

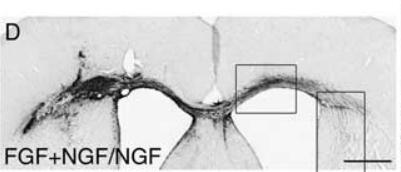

$\mathrm{N}$

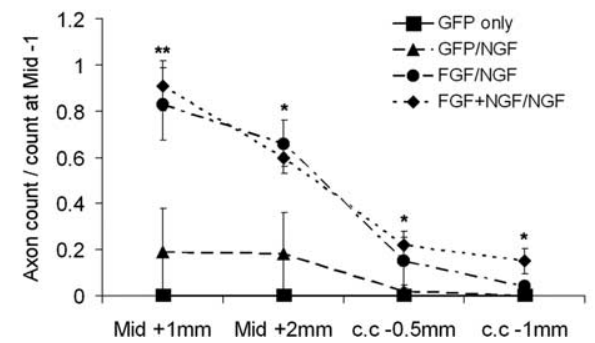

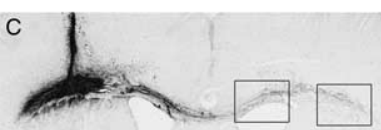

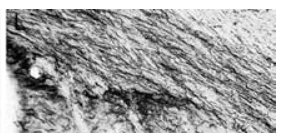

there was a needle track from an injection that included Ad-NGF; that is, every needle track in the combination FGF2/NGF pathways, and only the target needle track in GFP pathways (Fig. $7 B-M$ ). Growth within the corpus callosum was limited, but in animals with FGF2/NGF pathways, five of six had some callosal axon growth all the way across and into the target area (a distance of $\sim 5 \mathrm{~mm}$ ). Only one of three GFP-pathway animals had growth that far in the corpus callosum; in the other two, axons stopped within $1.5 \mathrm{~mm}$ of the transplant. Axon counts within the corpus callosum were compared by Mann-Whitney $U$ tests and found to be significantly higher at every path point when FGF2 or FGF2/ NGF were expressed in the corpus callosum, compared with GFP-pathway animals $(p<0.05)$. When growth within the corpus callosum was compared between animals with a cortical NGF target and those with a striatal NGF target, the pathways leading to a striatal target contained significantly more CGRP + fibers at midline $+1 \mathrm{~mm}$, but growth factor-expressing pathways contained more fibers than GFP controls in both experiments (Fig. $7 N$ ). The number of CGRP+ axons reaching each NGF-expressing target area (striatal or cortical) was similar, but with cortical targets, no difference existed between animals expressing FGF2 along the corpus callosum and those expressing only GFP (Fig. 7O). Obviously, many of the axons had followed an alternative pathway to the target, growing along the brain surface and avoiding the white matter of the corpus callosum altogether. To be sure that the CGRP+ fibers observed in the meninges were not from NGF-induced sprouting of endogenous nociceptive fibers, three control animals received the same Ad-FGF2/NGF pathway injections and Ad-NGF cortical targets, but no DRG cell transplants. In

these animals, there were no visible CGRP + fibers at the brain
surface or penetrating the cortex at any point after the same pe-

ing the needle track from the Ad-NGF target injection, sometimes in greater numbers than those turning toward the striatum. This is consistent with observations by Davies et al. (1997), who described axon growth out of the corpus callosum into the host prefrontal cortex as a default pathway for transplanted CGRP+ DRG neurons. With this in mind, we altered the adenovirus injection scheme to see whether greater numbers of fibers would choose that route if the NGF-expressing target was in the cortex rather than in the striatum. Pathways were constructed with either a combination of Ad-FGF2 and Ad-NGF (5:1) or Ad-GFP leading to the cortical NGF target (Fig. 7A). It was expected that with FGF2/NGF pathways, axons would extend along the corpus callosum from the transplant, as before, and then turn up to reach the target area on the contralateral side. Instead, the cortical expression of NGF greatly enhanced growth of CGRP + fibers along the brain surface in the meninges (Fig. $7 F, K$ ). Axons from these neurons grew upward along the transplant needle track and then along the brain surface, with some growing down again wherever riod of time (data not shown). The results of this experiment underscore the fact that virus-mediated expression of growth factors may increase growth to a particular target region, but endogenous cues in the environment of the host CNS also play a key role to influence pathway decisions.

\section{Discussion}

Stem cells have introduced a new enthusiasm for neuronal replacement strategies in chronic neurodegenerative disorders; however, the lack of appropriate environmental cues may prevent long distance axon growth to discrete target locations to reconstruct lost circuits. With this in mind, we set forth to evaluate the possibility of establishing crude guidance pathways to direct the growth of axons along a white matter pathway and turning toward a specified target in the gray matter. Although different neuronal populations would require different guidance 
pathways, CGRP + DRG neurons were selected because of the general knowledge of factors that influence their developmental growth, regeneration, and sprouting. The results confirmed the ability of FGF2 alone or in combination with NGF, but not L1CAM or TGF- $\beta 1$, to produce a growth promoting pathway extending from the DRG transplant to an NGF striatal target. To efficiently induce turning, strategic placement of both growth promoting factors and chemorepulsive factors led to a significant enhancement in axon growth and targeting along the pathway. Thus as with developmental guidance, similar attractive and repulsive cues can be used to enhance targeting of axons in the adult CNS. In general, this model could be easily adapted to investigate potential developmental guidance cues for circuit reconstruction of select neuronal populations within the adult brain.

During embryonic development, growth cones on different sensory neuronal subtypes respond to different guidance cues, depending on which receptors they express. Based on both in vitro and in vivo experiments, it has been determined that NGF acts as a chemoattractant for embryonic sensory axons expressing the highaffinity receptor tyrosine kinase Trk A (Gundersen and Barrett, 1979; Paves and Saarma, 1997; Tucker et al., 2001). In the adult, a subpopulation of these neurons retains their Trk A expression and alters their axon growth patterns in response to NGF.

Previously, we showed that after dorsal root rhizotomies, lesioned axons that would normally not re-enter the spinal cord were stimulated to do so by virus-mediated NGF expression in the dorsal horn (Romero et al., 2001). This probably reflects the ability of neurotrophins to increase the intrinsic growth state of neurons and overcome inhibitory signals present in the adult CNS environment (Cai et al., 1999). Virus-mediated expression of FGF2 in the spinal cord had a similar regenerative effect on sensory afferents after dorsal root injury, resulting in growth into the cord, consistent with the known neurite growth-promoting effects of FGF-receptor activation (Williams et al., 1994; Romero et al., 2001). Based on these lines of evidence, we chose NGF and FGF2 as our positive growth cues for transplanted postnatal DRG neurons in the adult rat corpus callosum, and both molecules improved axon growth and targeting. In opposition to these factors, we previously identified L1-CAM to inhibit NGF mediate sprouting or regeneration of these axons (Romero et al., 2001; Chauhdry et al., 2006), most likely through interactions with the semaphorin 3A receptor complex (Castellani et al., 2000; Chaudhry et al., 2006). Likewise, previous studies have show TGF- $\beta 1$ to be a key inducer of chondroitin sulfate proteoglycans by astrocytes (Asher et al., 2000; Smith and Strutz, 2005) and, thus, increases the general axonal growth inhibitory nature of the pathway (Snow et al., 1990; McKeon et al., 1991). Both of these factors showed dramatically less axon growth from DRG transplants than LacZ controls and acted as negative regulators of axon growth along the pathway.
Consistent axon growth away from the transplant site and along the white matter tract was only observed with injections of adenovirus encoding FGF2 or a combination of FGF2 and NGF along the corpus callosum. With Ad-GFP injections along the path and no NGF-expressing target in the contralateral hemisphere, no CGRP + fibers grew as far as the midline. In the presence of a NGF target only one of three animals had contralateral fiber growth along the connecting GFP pathway. This might have been from diffusion of NGF into the lateral ventricles which would sometimes direct axons out of the callosal pathway and along the ventricular surface (Fig. $3 B, D$ ). These results differ from those of Davies et al. (1997), who demonstrated longdistance growth of CGRP+ fibers within the adult corpus callosum after DRG transplants without accompanying overexpression of growth-supportive molecules. The discrepancy is most likely attributable to a difference in transplantation technique. Whereas we used a 30-guage needle for virus injections and transplants, they used a microtransplantation method to minimize glial scarring (Davies et al., 1997). For eventual clinical applications, it would be best to reduce the amount of tissue damage caused by the cell transplantation procedure because resultant scarring leads to expression of CSPGs which are known to interfere with axon regeneration (Davies et al., 1999; Jones et al., 2003; Silver and Miller, 2004). In this model, however, we demonstrate the effectiveness of preformed growth pathways in directing axon growth from transplants despite inhibitory signals present in the 


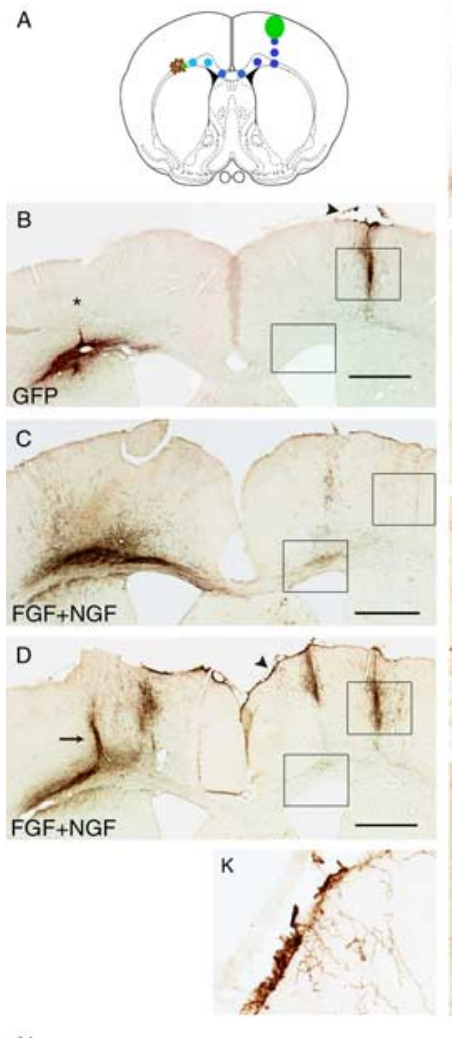

N

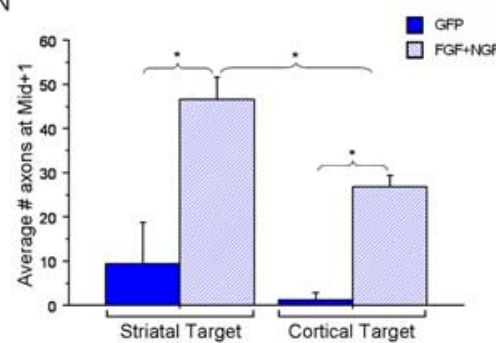

G
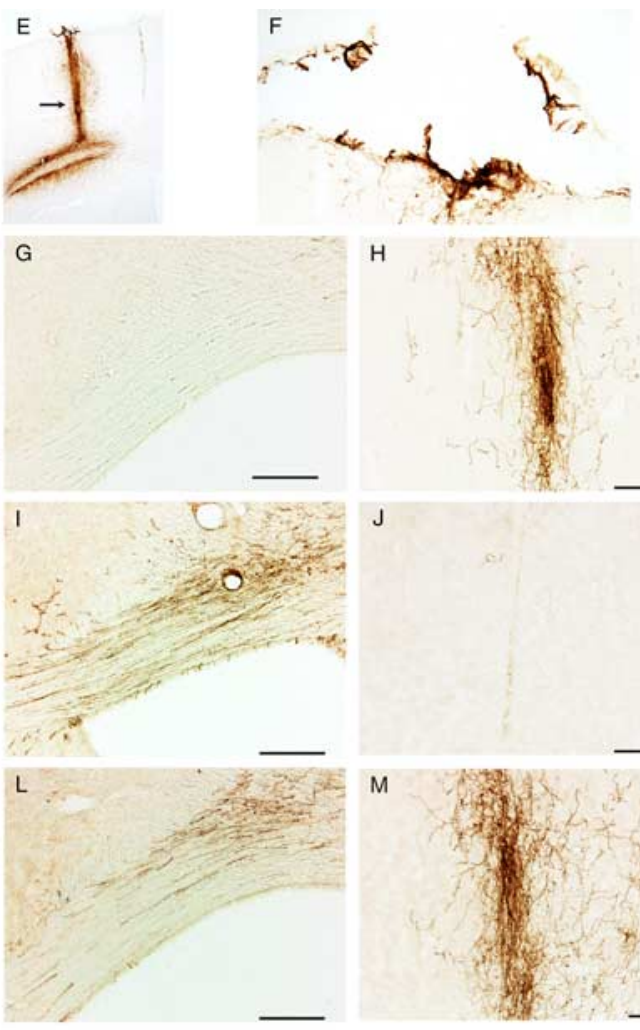

$\mathrm{O}$

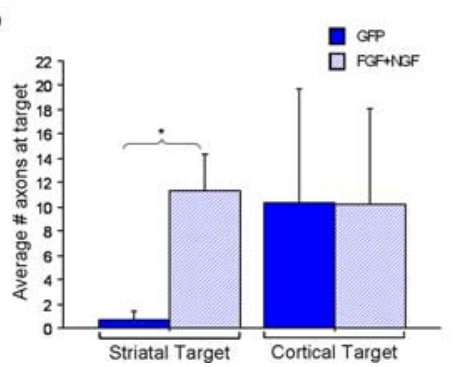

Figure 7. NGF overexpression in the cortex leads to axon growth along the brain surface when neurons are transplanted into the corpus callosum. $A$, Schematic of injection protocol with cortical target: Blue circles along corpus callosum represent Ad-FGF2/ NGF or Ad-GFP (control) injections, green circles represent Ad-NGF injections at the DRG transplant site (left) and cortical target (right). Brown circles represent transplanted DRG neurons, injected 1 week after the adenoviral pathway. $\boldsymbol{B}-\boldsymbol{M}$, Representative coronal sections after 3 weeks' growth, immunostained for CGRP. B, GFP pathway, NGF target in cortex; an asterisk indicates the area where axons are found growing up toward the brain surface in a separate section, shown in $E$; the arrowhead points to CGRP + fibers growing within the meninges, which have mostly pulled away from the brain parenchyma during tissue processing. C, D, FGF2+NGF (5:1) combination pathways, NGF target in cortex. Two different animals from this treatment group are represented to illustrate variability in axon-pathfinding decisions. The arrow in $\boldsymbol{D}$ points to CGRP + axons growing up toward the brain surface, whereas the arrowhead in $\boldsymbol{D}$ points out dense fiber growth within the meninges. $\boldsymbol{E}$, A separate section from the same animal as $\boldsymbol{B}$, showing where the CGRP + axons have grown up from the transplant site toward the brain surface. $\boldsymbol{F}$, Higher magnification of area indicated by arrowhead in $\boldsymbol{B}$, showing CGRP + fiber growth within the meninges. $\boldsymbol{G}, \boldsymbol{H}$, Higher magnification of boxed areas in $\boldsymbol{B}$. $\boldsymbol{I}, \boldsymbol{J}$, Higher magnification of boxed areas in $\boldsymbol{C}$. $\boldsymbol{K}$, Higher magnification of area indicated by arrowhead in $\boldsymbol{D}$, showing CGRP + fibers within the pia and coursing down into the cortex. $\boldsymbol{L}, \boldsymbol{M}$, Higher magnification of boxed areas in $\boldsymbol{D}$. $\boldsymbol{N}$, Quantification of CGRP + axon growth in control (GFP) pathways and FGF2 + NGF pathways at $1 \mathrm{~mm}$ beyond the midline when the NGF-expressing target was either in the striatum or in the cortex. There is significantly more axon growth in FGF2 + NGF pathways than in GFP pathways for both injection paradigms, but fewer axons follow the corpus callosum pathway when the target is in the cortex because they choose the alternative route along the brain surface. $\mathbf{0 , 0 u a n t i f i c a t i o n ~ o f ~ C G R P ~}+$ axon growth into the target area, either halfway between the brain surface and the corpus callosum (pictured in $\boldsymbol{H}, \boldsymbol{J}$, and $\boldsymbol{M}$ ), or $0.5 \mathrm{~mm}$ below the corpus callosum in the striatum. With the striatal target, there is a significant difference between GFP pathways and FGF2 + NGF pathways, but with the cortical target, axon growth along the brain surface to the target eliminates the difference between pathways. Error bars represent means \pm SEM. Mann-Whitney $U$ tests at each path point. ${ }^{*} p<0.05$. GFP/NGF, $n=3$; FGF + NGF/NGF, $n=4$. Scale bars: $\boldsymbol{B}-\boldsymbol{D}, 1 \mathrm{~mm} ; \boldsymbol{E}-\boldsymbol{J}, 200 \mu \mathrm{m}$.

host tissue, which may be a factor when transplants are made into previously injured brains or spinal cords.

We next sought to increase the proportion of axons that turned toward the target rather than continuing along the exter-

nal capsule or growing up toward the cortex by expressing a chemorepulsive molecule, semaphorin $3 \mathrm{~A}$, dorsolateral to the turn. An extra stimulus is necessary in this situation because white matter may support axon growth that is parallel to, but not perpendicular to the tract, and crossover between white and gray matter is usually restricted by this cytoarchitecture (Crutcher, 1989; Pettigrew and Crutcher, 1999). Even though, priming neurons with either NGF or cAMP reduced the parallel orientation of growing axons to myelin (Pettigrew and Crutcher, 2001), NGF alone only supported the turning out of white matter for about half the axons. Our choice of molecules for this purpose was again based on knowledge of sensory neuron development. Centrally projecting nociceptive DRG axons are prevented from overshooting their destination in the dorsalmost laminas of the embryonic spinal cord by expression of semaphorin $3 \mathrm{~A}$ in the ventral cord. (Messersmith et al., 1995). Peripheral sensory axons also have their growth pathways restricted by the presence of semaphorin $3 \mathrm{~A}$ in surrounding, nontarget mesenchymal tissue, and both central and peripheral semaphorin expression patterns are spatially and temporally regulated to coincide with the development of sensory nerve tracts (Giger et al., 1996; Masuda and Shiga, 2005). Adult nociceptive sensory fibers are similarly repelled and prevented from sprouting by semaphorin 3A (Tanelian et al., 1997; Tang et al., 2004). Consistent with these findings, the expression of semaphorin $3 \mathrm{~A}$ dorsal and lateral to the desired ventral turn in the guidance pathway increased the proportion of axons making the "correct" turning decision from 51 to $77 \%$. Whereas expression of positive growth cues along a desired pathway helps to target axon growth, negative cues in the periphery prevent growth into nontarget areas and thus improve accuracy in our model as in embryonic development.

An interesting observation in initial experiments was the growth of some CGRP+ axons from the corpus callosum up into the overlying cortex along the needle track used to make Ad-NGF target injections. Although the Ad-NGF was injected into the striatum, it is likely that a small amount of virus was pulled up the track as the needle was being withdrawn from the brain, leading to some cortical NGF expression. This small amount of expression was apparently enough to encourage growth in that direction, possibly because the host environment in the cortex is inherently more growthpermissive than the striatum. In fact, previous DRG transplants into the corpus callosum by Davies et al. (1997) resulted in a few CGRP+ 
fibers turning up into the host cortex without any induced expression of neurotrophic factors. Based on these observations, we altered the pathway-injection scheme to place the NGF target in the cortex contralateral to the transplantation site. Our prediction was that axons would grow along the corpus callosum as before, and then the majority of the fibers would turn up toward the target. Instead, the high cortical NGF expression directed most axon growth from the transplant site up to and along the brain surface, with some fibers diving down into cortical needle tracks where Ad-NGF had been injected. The combination of NGF availability and the highly vascular nature of the leptomeninges may have led to this pathway decision, because endothelial basement membranes are a rich source of laminin. Laminin, by way of integrin receptors, activates signaling pathways that overlap downstream with NGF signaling pathways, leading to further enhanced neurite outgrowth from sensory neurons (Liu et al., 2002; Tucker et al., 2005). In addition, these axons follow a path of least resistance (Ramon y Cajal, 1928), in which there are less growth constraints for axons growing within the relative openness of the subarachnoid space than within the densely myelinated corpus callosum. The results of this experiment highlight the importance of endogenous growth cues in the host environment for determining axon trajectories of transplanted neurons.

The current study provides evidence that preformed guidance pathways created by injection of viral vectors can direct axon growth from transplanted neurons to desired target locations. Furthermore, the accuracy of targeting may be improved by judicious expression of chemorepulsive molecules in surrounding areas. This technique could potentially improve outcomes in therapeutic transplantation paradigms requiring axon growth over long distances and/or through inhibitory host tissue.

\section{References}

Asher RA, Morgenstern DA, Fidler PS, Adcock KH, Oohira A, Braistead JE, Levine JM, Margolis RU, Rogers JH, Fawcett JW (2000) Neurocan is upregulated in injured brain and in cytokine-treated astrocytes. J Neurosci 20:2427-2438.

Aubert I, Ridet JL, Gage FH (1995) Regeneration in the adult mammalian CNS: guided by development. Curr Opin Neurobiol 5:625-635.

Bjorklund A, Lindvall O (2000) Cell replacement therapies for central nervous system disorders. Nature Neuroscience 3:537-544.

Cai D, Shen Y, De Bellard M, Tang S, Filbin MT (1999) Prior exposure to neurotrophins blocks inhibition of axonal regeneration by MAG and Myelin via a cAMP-dependent mechanism. Neuron 22:89-101.

Cai D, Qiu J, Cao Z, McAtee M, Bregman BS, Filbin MT (2001) Neuronal cyclic AMP controls the developmental loss in ability of axons to regenerate. J Neurosci 21:4731-4739.

Castellani V, Chedotal A, Schachner M, Faivre-Sarrailh C, Rougon G., (2000) Analysis of the L1-eficient mouse phenotype reveals cross-talk between Sema3A and L1 signaling pathways in axonal guidance. Neuron 27:237-249.

Chaudhry N, de Silva U, Smith GM (2006) Cell adhesion molecule L1 modulates nerve-growth-factor-induced CGRP-IR fiber sprouting. Exp Neurol 202:238-249.

Crutcher KA (1989) Tissue sections from the mature rat brain and spinal cord as substrates for neurite outgrowth in vitro: extensive growth on gray matter but little growth on white matter. Exp Neurol 104:39-54.

Davies SJ, Field PM, Raisman G (1994) Long interfascicular axon growth from embryonic neurons transplanted into adult myelinated tracts. J Neurosci 14:1596-1612.

Davies SJ, Fitch MT, Memberg SP, Hall AK, Raisman G, Silver J (1997) Regeneration of adult axons in white matter tracts of the central nervous system. Nature 390:680-683.

Davies SJ, Goucher DR, Doller C, Silver J (1999) Robust regeneration of adult sensory axons in degenerating white matter of the adult rat spinal cord. J Neurosci 19:5810-5822.

Domeniconi M, Filbin MT (2005) Overcoming inhibitors in myelin to promote axonal regeneration. J Neurological Sci 233:43-47.
Giger R, Wolfer D, De Wit G, Verhaagen J (1996) Anatomy of rat semaphorin III/collapsin-1 mRNA expression and relationship to developing nerve tracts during neuroembryogenesis. J Comp Neurol 375:378-392.

Goldberg JL, Klassen MP, Hua Y, Barres BA (2002) Amacrine-signaled loss of intrinsic axon growth ability by retinal ganglion cells. Science 296:1860-1864.

Gundersen R, Barrett JN (1979) Neuronal chemotaxis: chick dorsal root axons turn toward high concentrations of nerve growth factor. Science 206:1079-1080.

He TC, Zhou S, da Costa LT, Yu J, Kinzler KW, Vogelstein B (1998) A simplified system for generating recombinant adenoviruses. Proc Natl Acad Sci USA 95:2509-2514.

Isacson O, Bjorklund LM, Schumacher JM (2003) Toward full restoration of synaptic and terminal function of the dopaminergic system in Parkinson's disease by stem cells. Ann Neurol 53:S135-S148.

Jones LL, Sajed D, Tuszynski MH (2003) Axonal regeneration through regions of chondroitin sulfate proteoglycan deposition after spinal cord injury: a balance of permissiveness and inhibition. J Neurosci 23:9276-9288.

Liu RY, Schmid RS, Snider WD, Maness PF (2002) NGF Enhances Sensory Axon Growth Induced by Laminin but Not by the L1 Cell Adhesion Molecule. Mol Cell Neurosci 20:2-12.

Masuda T, Shiga T (2005) Chemorepulsion and cell adhesion molecules in patterning initial trajectories of sensory axons. Neurosci Res 51:337-347.

McKeon RJ, Schreiber RC, Rudge JS, Silver J (1991) Reduction of neurite outgrowth in a model of glial scarring following CNS injury is correlated with expression of inhibitory molecules on reactive astrocytes. J Neurosci 11:3398-3411.

Messersmith EK, Leonardo ED, Shatz CJ, Tessier-Lavigne M, Goodman CS, Kolodkin AL (1995) Semaphorin III can function as a selective chemorepellent to pattern sensory projections in the spinal cord. Neuron 14:949-959.

Paves H, Saarma M (1997) Neurotrophins as in vitro growth cone guidance molecules for embryonic sensory neurons. Cell Tissue Res 290:285-297.

Pettigrew DB, Crutcher KA (1999) White matter of the CNS supports or inhibits neurite outgrowth in vitro depending on geometry. J Neurosci 19:8358-8366.

Pettigrew DB, Crutcher KA (2001) Myelin contributes to the parallel orientation of axonal growth on white matter in vitro. BMC Neurosci 2:9.

Ramón y Cajal S (1928) Degeneration and regeneration in the nervous system. New York: Haffner.

Romero MI, Smith GM (1998) Adenoviral gene transfer into the normal and injured spinal cord: enhanced transgene stability by combined administration of temperature-sensitive virus and transient immune blockade. Gene Ther 5:1612-1621.

Romero MI, Rangappa N, Li L, Lightfoot E, Garry MG, Smith GM (2000) Extensive sprouting of sensory afferents and hyperalgesia induced by conditional expression of nerve growth factor in the adult spinal cord. J Neurosci 20:4435-4445.

Romero MI, Rangappa N, Garry MG, Smith GM (2001) Functional regeneration of chronically injured sensory afferents into adult spinal cord after neurotrophin gene therapy. J Neurosci 21:8408-8416.

Sandvig A, Berry M, Barrett LB, Butt A, Logan A (2004) Myelin-, reactive glia-, and scar-derived CNS axon growth inhibitors: expression, receptor signaling, and correlation with axon regeneration. Glia 46:225-251.

Schwab JM, Bernard F, Moreau-Fauvarque C, Chedotal A (2005) Injury reactive myelin/oligodendrocyte-derived axon growth inhibition in the adult mammalian central nervous system. Brain Res Rev 49:295-299.

Silver J, Miller JH (2004) Regeneration beyond the glial scar. Nat Rev Neurosci 5:146-156.

Tanelian DL, Barry MA, Johnston SA, Le T, Smith GM (1997) Semaphorin III can repulse and inhibit adult sensory afferents in vivo. Nat Med 3:1398-1401.

Tang XQ, Tanelian DL, Smith GM (2004) Semaphorin3A inhibits nerve growth factor-induced sprouting of nociceptive afferents in adult rat spinal cord. J Neurosci 24:819-827.

Tucker BA, Rahimtula M, Mearow KM (2005) Integrin activation and neurotrophin signaling cooperate to enhance neurite outgrowth in sensory neurons. J Comp Neurol 486:267-280.

Tucker KL, Meyer M, Barde YA (2001) Neurotrophins are required for nerve growth during development. Nat Neurosci 4:29-37.

Williams EJ, Furness J, Walsh FS, Doherty P (1994) Activation of the FGF receptor underlies neurite outgrowth stimulated by L1, N-CAM, and N-cadherin. Neuron 13:583-594. 